\title{
PHYSICAL CONDITIONS IN MOLECULAR CLOUDS IN THE ARM AND INTERARM REGIONS OF M51
}

\author{
Jin Koda $^{1}$, Nick Scoville ${ }^{2}$, Tetsuo Hasegawa ${ }^{3}$, Daniela Calzetti ${ }^{4}$, Jennifer Donovan Meyer $^{1}$, Fumi Egusa ${ }^{5}$, \\ Robert Kennicutt ${ }^{6}$, Nario Kuno ${ }^{7,8}$, Melissa Louie ${ }^{1}$, Rieko Momose $^{9}$, Tsuyoshi Sawada ${ }^{3,10}$, \\ KAZUO SORAI ${ }^{11}$, AND MichiKo UMEI ${ }^{11}$ \\ ${ }^{1}$ Department of Physics and Astronomy, Stony Brook University, Stony Brook, NY 11794-3800, USA; jin.koda@ stonybrook.edu \\ ${ }_{2}^{2}$ Department of Astronomy, California Institute of Technology, Pasadena, CA 91125, USA \\ ${ }^{3}$ National Astronomical Observatory of Japan, NAOJ Chile Observatory, Joaquín Montero 3000 Oficina 702, Vitacura, Santiago 763-0409, Chile \\ ${ }^{4}$ Department of Astronomy, University of Massachusetts, Amherst, MA 01003, USA \\ ${ }^{5}$ Department of Space Astronomy and Astrophysics, Institute of Space and Astronautical Science, Japan Aerospace Exploration Agency, Japan \\ ${ }^{6}$ Institute of Astronomy, University of Cambridge, Cambridge CB3 OHA, UK \\ ${ }^{7}$ Nobeyama Radio Observatory, Minamimaki, Minamisaku, Nagano, 384-1305, Japan \\ ${ }^{8}$ The Graduate University for Advanced Studies (SOKENDAI), 2-21-1 Osawa, Mitaka, Tokyo 181-0015, Japan \\ ${ }^{9}$ Institute for Cosmic Ray Research, University of Tokyo, 5-1-5 Kashiwa-no-Ha, Kashiwa City, Chiba, 277-8582, Japan \\ ${ }^{10}$ Joint ALMA Office, Alonso de Córdova 3107, Vitacura, Santiago 763-0355, Chile \\ ${ }^{11}$ Department of Physics/Department of Cosmosciences, Hokkaido University, Kita-ku, Sapporo 060-0810, Japan \\ Received 2012 April 20; accepted 2012 October 22; published 2012 November 20
}

\begin{abstract}
We report systematic variations in the emission line ratio of the CO $J=2-1$ and $J=1-0$ transitions $\left(R_{2-1 / 1-0}\right)$ in the grand-design spiral galaxy M51. The $R_{2-1 / 1-0}$ ratio shows clear evidence for the evolution of molecular gas from the upstream interarm regions into the spiral arms and back into the downstream interarm regions. In the interarm regions, $R_{2-1 / 1-0}$ is typically $<0.7$ (and often $0.4-0.6$ ); this is similar to the ratios observed in Galactic giant molecular clouds (GMCs) with low far-infrared luminosities. However, the ratio rises to $>0.7$ (often 0.8-1.0) in the spiral arms, particularly at the leading (downstream) edge of the molecular arms. These trends are similar to those seen in Galactic GMCs with OB star formation (presumably in the Galactic spiral arms). $R_{2-1 / 1-0}$ is also high, $\sim 0.8-1.0$, in the central region of M51. Analysis of the molecular excitation using a Large Velocity Gradient radiative transfer calculation provides insight into the changes in the physical conditions of molecular gas between the arm and interarm regions: cold and low-density gas $\left(\lesssim 10 \mathrm{~K}, \lesssim 300 \mathrm{~cm}^{-3}\right)$ is required for the interarm GMCs, but this gas must become warmer and/or denser in the more active star-forming spiral arms. The ratio $R_{2-1 / 1-0}$ is higher in areas of high $24 \mu \mathrm{m}$ dust surface brightness (which is an approximate tracer of star formation rate surface density) and high $\mathrm{CO}(1-0)$ integrated intensity (i.e., a well-calibrated tracer of total molecular gas surface density). The systematic enhancement of the $\mathrm{CO}(2-1)$ line relative to $\mathrm{CO}(1-0)$ in luminous star-forming regions suggests that some caution is needed when using $\mathrm{CO}(2-1)$ as a tracer of bulk molecular gas mass, especially when galactic structures are resolved.
\end{abstract}

Key words: evolution - galaxies: individual (NGC 5194, M51) - ISM: clouds - ISM: molecules

Online-only material: color figures

\section{INTRODUCTION}

The standard, albeit simplistic, picture of interstellar matter (ISM) phases posits that giant molecular clouds (GMCs) are assembled in spiral arm shocks from diffuse interarm Hi gas and then photodissociated back into the atomic phase by OB star formation within the spiral arms. This picture predicts a rapid gas-phase change across spiral arms from atomic to molecular and back into the atomic after spiral arm passage. New $\mathrm{CO}(1-0)$ observations of M51 suggest a very different picture in which there is little (or no) gas-phase change between interarm and arm regions, with the majority $(70 \%-80 \%)$ of the neutral gas remaining molecular from arm entry through the interarm region and into the next spiral arm passage (Koda et al. 2009). The molecular gas fraction could be even higher if dark $\mathrm{H}_{2}$ gas exists (e.g., Planck Collaboration et al. 2011; Grenier et al. 2005), which is not detectable in $\mathrm{CO}$ emission. In this new picture, the obvious molecular spiral arms are assembled from pre-existing small GMCs by spiral arm forcing and orbit crowding within the arms. A fraction of the gas forms dense cores in the molecular spiral arms, leading to star formation (Egusa et al. 2011), but the majority is shredded apart by spiral shearing motions and goes back to the interarm regions as smaller GMCs (Koda et al. 2009; Wada \& Koda 2004).

Questions still remain as to what the physical conditions of the abundant molecular gas in the interarm regions are and how these conditions change within the spiral arms. The lowest rotational transition $J=1-0$ of the carbon monoxide (CO) molecule is the most reliable tracer of the overall molecular gas mass and surface density in galaxies. The transition energy of the $\mathrm{CO}(1-0)$ corresponds to $E / k \sim 5.5 \mathrm{~K}$, and this is conveniently below the typical observed temperatures of resolved GMCs ( $\sim 10 \mathrm{~K}$; Scoville \& Sanders 1987); in addition, the critical density for $\mathrm{CO}(1-0)$ collisional excitation in the presence of photon trapping is a few $\times 100 \mathrm{H}_{2} \mathrm{~cm}^{-3}$, which is roughly the same as the average density within the GMCs (Solomon et al. 1987). Therefore, $\mathrm{CO}(1-0)$ line emission arises from the levels that are close to thermalized, and the $\mathrm{CO}(1-0)$ luminosity traces their bulk molecular mass (see Scoville \& Sanders 1987). Empirically, $\mathrm{CO}(1-0)$ luminosities of GMCs are correlated with their virial mass estimates. A nearly constant mass-to-light ratio has been calibrated with virial masses of resolved GMCs in the Galaxy (Solomon et al. 1987; Scoville \& Sanders 1987) and in nearby spiral galaxies (Bolatto et al. 2008; Donovan Meyer 
et al. 2012). (Note that, despite the constant ratio observed in normal spiral galaxies, there are discussions on its variations in low-metallicity dwarf galaxies (Israel 1997; Leroy et al. 2009a), where self-shielding of GMCs from ambient ultraviolet radiation may not be as effective.)

Within the GMCs only a small fraction of the gas is typically within the dense core regions leading to star formation. $\mathrm{HCN}(1-0)$, having a higher critical density $\left(\sim 10^{5} \mathrm{~cm}^{-3}\right.$; Kohno et al. 1996) than low-J CO lines, traces the emission from such dense cores, and this line emission exhibits a linear correlation with infrared (IR) emission, a tracer of embedded star formation, over 7-8 orders of magnitude-from starburst galaxies down to small dense cores within Galactic GMCs (Gao \& Solomon 2004; Wu et al. 2005). Recently, CO(3-2) has also been used to trace dense cores (Muraoka et al. 2007; Wilson et al. 2009). Its higher critical density $\left(\gtrsim 10^{4} \mathrm{~cm}^{-3}\right)$ and relatively high temperature corresponding to the upper energy level $(\sim 33 \mathrm{~K})$ imply that it traces the environments with active star formation-either dense star-forming molecular cores or gas heated by recent star formation. This has been demonstrated by Komugi et al. (2007), who found a linear correlation between surface densities of $\mathrm{CO}(3-2)$ emission and star formation in nearby galaxies.

$\mathrm{CO}(2-1)$ emission has an upper-level energy temperature of $\sim 16.5 \mathrm{~K}$ and a collisional critical density of $\sim 10^{3-4} \mathrm{~cm}^{-3}$, slightly above the standard GMC temperature and density; hence, the $\mathrm{CO}(2-1) / \mathrm{CO}(1-0)$ line ratio $\left(R_{2-1 / 1-0}\right)$ can trace the change of physical conditions of the bulk molecular gas. In fact, the ratio shows a systematic variation in the Galaxy (Sakamoto et al. 1999) and has been used to investigate the varying physical conditions (Sakamoto et al. 1997; Sawada et al. 2001). $R_{2-1 / 1-0}$ has also been used to infer gas physical conditions in external galaxies, especially in their central regions (Knapp et al. 1980; Braine \& Combes 1992; Turner et al. 1993; Aalto et al. 1995; Harrison et al. 1999).

Attempts to map $R_{2-1 / 1-0}$ across galaxy disks have remained inconclusive. Garcia-Burillo et al. (1993) analyzed their 2-1 and 1-0 imaging of M51 and found no systematic variation in $R_{2-1 / 1-0}$ between the spiral arm and interarm regions. However, the early $\mathrm{CO}(1-0)$ data (Nakai et al. 1994) used in their analysis appear to be inconsistent by a factor of $\sim 2$ in flux with four other later measurements (Koda et al. 2011), perhaps due to calibration difficulties 20 years ago. Schinnerer et al. (2010) analyzed $R_{2-1 / 1-0}$, as well as other molecular lines, in the western spiral arm of M51 using data obtained with interferometers, which was unfortunately combined with the old Nakai et al. (1994) single-dish data with the apparent calibration problem. Therefore, the question of large-scale variation of $R_{2-1 / 1-0}$ remains unanswered. Here, we present new $\mathrm{CO}(2-1)$ and $\mathrm{CO}(1-0)$ imaging with a new and consistent observation technique and careful calibration for both lines. These new data permit much-improved measurements of the spatial variations in $R_{2-1 / 1-0}$ in both arm and interarm regions of M51.

\section{CO AND INFRARED DATA}

The CO $J=1-0$ data are taken from Koda et al. (2011), which were taken as a part of the CARMA NObeyama Nearbygalaxies (CANON) survey. The original observations include both interferometer data from the Combined Array for Research in Millimeter Astronomy (CARMA) and single-dish data from the Nobeyama Radio Observatory $45 \mathrm{~m}$ telescope (NRO45). However, we use only the NRO45 data here to avoid unnecessary concerns, if any, about potential imaging artifacts introduced in the process of image reconstruction. CARMA data, observing small angular scales, do not play a role in our analysis, since we use a spatial resolution of $19^{\prime \prime} .7$.

The improved accuracy of the new data is due to the 25-BEam Array Receiver System (BEARS; Sunada et al. 2000) and the On-The-Fly (OTF) mapping technique (Sawada et al. 2008). They enable us to map the large galaxy within a short observing duration, ensuring consistency in calibration. Multiple OTF scans in the R.A. and decl. directions are averaged to minimize systematic errors, which appear dominantly along the scan directions (note that this type of error could not be characterized without the OTF technique). Therefore, the new data provide a much higher accuracy in relative calibration across the map. The absolute flux calibration is consistent with other independent measurements at a few percent level. The details of the data reduction are given in Koda et al. (2011). The final data cube has a spatial resolution of 19.7 (degraded by gridding from the beam size of $15^{\prime \prime}$ ) and a $1 \sigma$ noise of $\sim 37 \mathrm{mK}$ on the mainbeam temperature scale in a $10 \mathrm{~km} \mathrm{~s}^{-1}$ channel at a pixel size of 5 . $96 \times 5$ 5.96. It covers the entire molecular disks of NGC 5194 (main galaxy) and NGC 5195 (companion).

The CO $J=2-1$ data are from Schuster et al. (2007), which are distributed as a part of the HERA CO Line Extragalactic Survey (HERACLES; Leroy et al. 2009b). The data are taken with the 18 element focal plane heterodyne receiver array (HERA) on the IRAM $30 \mathrm{~m}$ telescope. The OTF technique was also employed. The 2-1 data cover the entire disk of NGC 5194 but do not include NGC 5195. The original integrated intensity image is in Schuster et al. (2007). The spatial resolution is 11", and the noise is $\sim 22 \mathrm{mK}$ in a $10 \mathrm{~km} \mathrm{~s}^{-1}$ channel on the mainbeam temperature scale. The $\mathrm{CO}(2-1)$ data cube was smoothed to the 19.7 resolution and regridded to the $\mathrm{CO}(1-0)$ reference grid. When comparing the two maps, we noticed that the global $\mathrm{CO}(2-1)$ distribution is offset by $\sim 1$ pixel in the southwest direction with respect to $\mathrm{CO}(1-0)$. We therefore shifted the $\mathrm{CO}(2-1)$ data correspondingly before making the integrated intensity maps with both lines integrated over the same velocity ranges referenced to the observed rotational velocity field in the galaxy. This treatment does not change the conclusions in this paper, as the spiral arms and interarm regions are considerably wider than one pixel.

The Spitzer $24 \mu \mathrm{m}$ image and $\mathrm{H} \alpha$ image are taken from the archive of the Spitzer Infrared Nearby Galaxies Survey (SINGS; Kennicutt et al. 2003). The Galaxy Evolution Explorer farultraviolet (FUV; $1539 \AA$ ) image (Gil de Paz et al. 2007) is taken through GalexView. We adopt the central coordinates of $(\alpha, \delta)_{\mathrm{J} 2000}=\left(13^{\mathrm{h}} 29^{\mathrm{m}} 52^{\mathrm{s}} .7,+47^{\mathrm{d}} 11^{\mathrm{m}} 42^{\mathrm{s}} .8\right)$ from Hagiwara et al. (2001).

\section{CO LINE RATIOS}

Line ratio images require high significance detections, especially of the denominator emission line; therefore, in the outer disk of NGC 5194 where the line brightness decreases, smoothing and/or clipping of low significance data are required. We adaptively smoothed the $\mathrm{CO}(1-0)$ data with the spatial binning method developed by Cappellari \& Copin (2003) and used data clipping in these areas when a large number of pixels $(>10)$ need to be averaged; identical smoothing and clipping were applied to the $\mathrm{CO}(2-1)$ map. Figures $1(\mathrm{a})$ and (b) show the resultant maps for $\mathrm{CO}(1-0)$ and $\mathrm{CO}(2-1)$.

The noise levels change across the maps as a result of the variable velocity widths for integration and adaptive smoothing, but the signal-to-noise ratios are high across the entire detected 

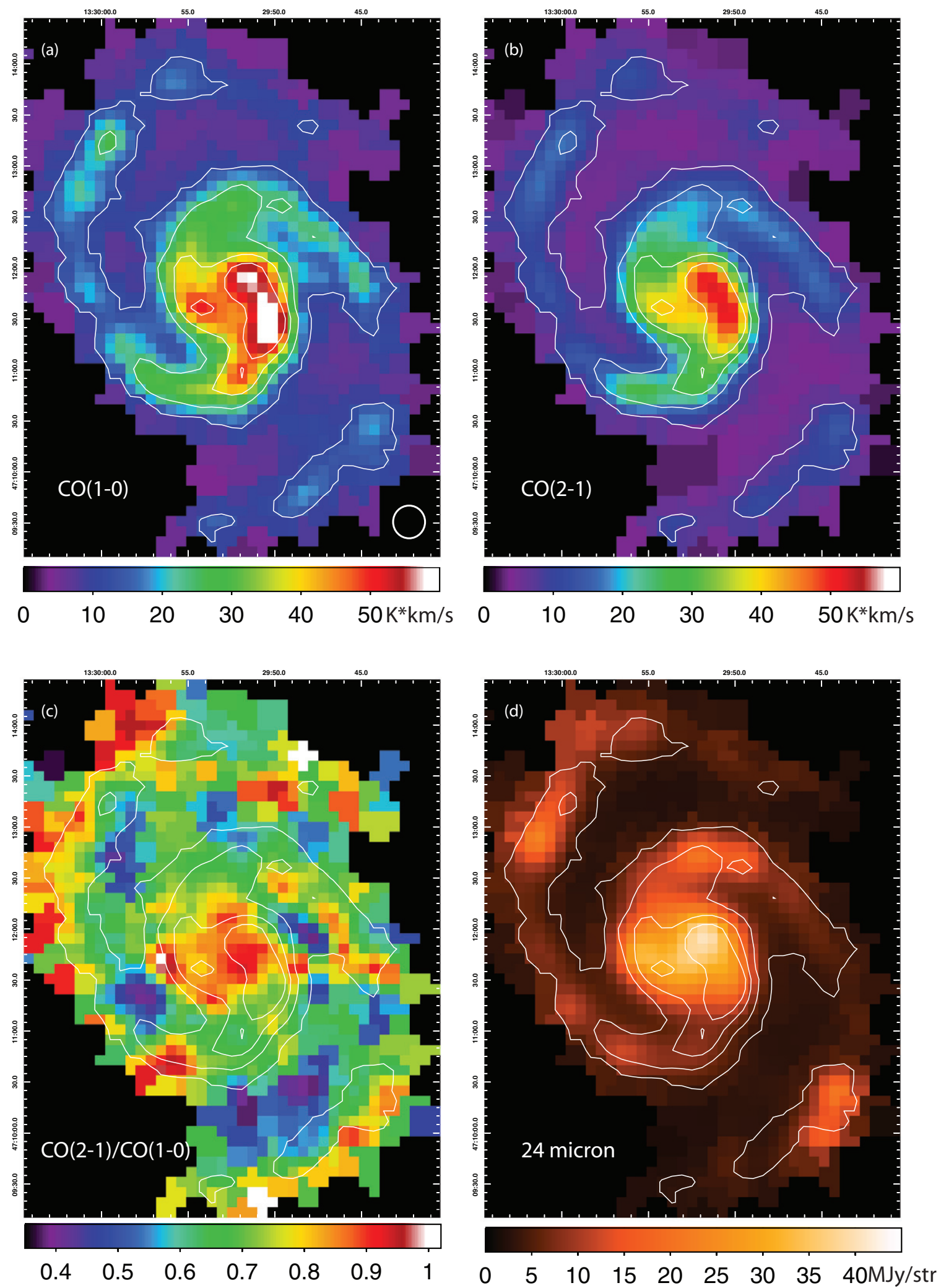

Figure 1. Maps of the central 5.4 $\times 4.1$ region of M51 in the J2000 coordinate system. (a) CO $J=1-0$ integrated intensity, $I_{\mathrm{CO}(1-0)}$, map in units of main-beam temperature. (b) $\mathrm{CO} J=2-1$ integrated intensity, $I_{\mathrm{CO}(2-1)}$, map in units of main-beam temperature. (c) $\mathrm{CO} J=2-1 / 1-0$ line ratio, $R_{2-1 / 1-0}\left(\equiv I_{\mathrm{CO}(2-1)} / I_{\mathrm{CO}(1-0)}\right)$, map. (d) Spitzer $24 \mu \mathrm{m}$ surface brightness, $f_{24} \mu \mathrm{m}$, image. The signal-to-noise ratios are high across the entire area, including the edges, i.e., $\gtrsim 10 \sigma$ in both CO(1-0) and $\mathrm{CO}(2-1)$. The circle at the bottom-right corner in (a) is the beam size of the $\mathrm{CO} J=1-0 \mathrm{map}, 19^{\prime \prime} .7$ ( $780 \mathrm{pc}$ at the distance of $8.2 \mathrm{Mpc}$ ). All other images are smoothed to this resolution. Contours in all panels are at $I_{\mathrm{CO}(1-0)}=12,24,36$, and $48 \mathrm{~K} \mathrm{~km} \mathrm{~s}^{-1}$. The line ratio varies systematically between the spiral arms and interarm regions.

(A color version of this figure is available in the online journal.) 

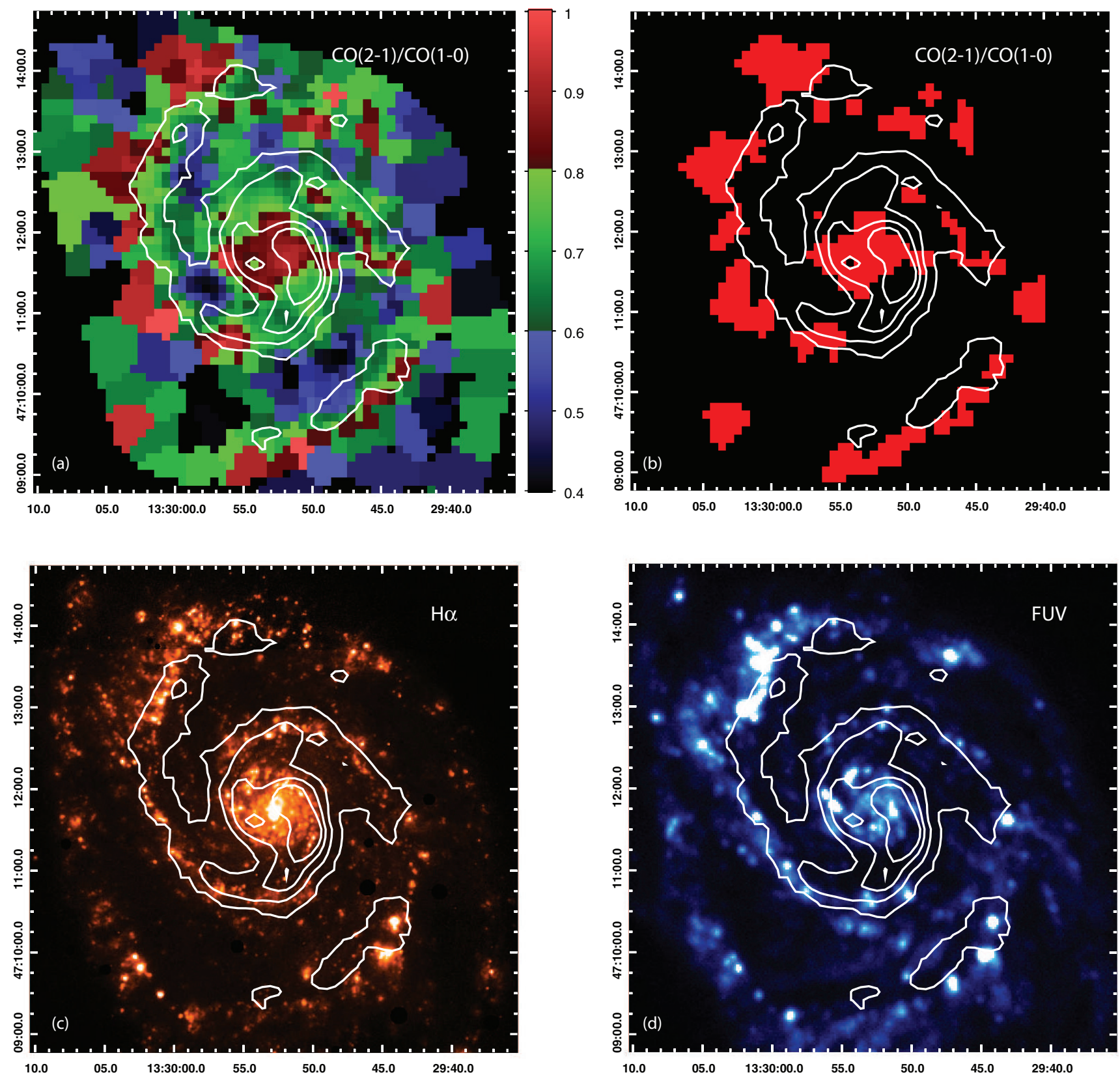

Figure 2. Locations of high and low $R_{2-1 / 1-0}$ with respect to the molecular spiral arms and star-forming regions. (a) Map of $R_{2-1 / 1-0}$. The same as Figure 1(c), but with a different color palette to separate the gas with $R_{2-1 / 1-0}<0.6,0.6-0.8$, and $>0.8$. This figure includes the outer areas where a large number of pixels ( $>10$ ) need to be averaged for reference. (b) Map of high $R_{2-1 / 1-0}(>0.8)$. (c) $\mathrm{H} \alpha$ image. (d) FUV image. Contours are the same as those in Figure 1 and are at $I_{\mathrm{CO}(1-0)}=$ $12,24,36$, and $48 \mathrm{~K} \mathrm{~km} \mathrm{~s}^{-1}$.

(A color version of this figure is available in the online journal.)

area, including at the edges, i.e., $\gtrsim 10 \sigma$ in both $\mathrm{CO}(1-0)$ and $\mathrm{CO}(2-1)$.

The $24 \mu \mathrm{m}$ image is processed in the same way and is shown in Figure 1(d). The $24 \mu \mathrm{m}$ emission appears at the leading (downstream) edges/sides of the $\mathrm{CO}(1-0)$ spiral arms (contours). Figures 2(c) and (d) show the $\mathrm{H} \alpha$ and FUV images at higher resolutions. These emissions, tracing recent star formation, are in general at the leading edges of the molecular spiral arms.

\subsection{Spatial Distribution}

Figure 1 (c) is the $R_{2-1 / 1-0}$ map and shows a spiral pattern of elevated $R_{2-1 / 1-0}$, i.e., $R_{2-1 / 1-0}$ varies systematically between the spiral arm and interarm regions. In the interarm regions, this ratio is $<0.7$ and often as small as $\sim 0.4-0.6$, while around the molecular spiral arms it is elevated to $>0.7$ and often becomes as high as $\sim 0.8-1.0$, mainly at the leading (downstream) edges of the arms (see also Figures 2(a) and (b)). The average $R_{2-1 / 1-0}$ over the entire disk is $\sim 0.70$. High ratios also appear occasionally at some upstream edges (e.g., in the northwest quadrant). We note again that both $\mathrm{CO}(1-0)$ and $\mathrm{CO}(2-1)$ are detected significantly even at the edge of this map. In the central $70^{\prime \prime}$ ( $\left.\sim 2.8 \mathrm{kpc}\right), R_{2-1 / 1-0}$ is also high, $\sim 0.8-1.0$. The high ratio appears to coincide with strong $24 \mu \mathrm{m}$ emission (Figure 1(d)). Figure 3 shows the phase diagrams of the $\mathrm{CO}(1-0)$ integrated intensity and $R_{2-1 / 1-0}$, again evidencing the association of the high ratio with the molecular spiral arms, especially at their leading edges/sides (i.e., right-hand side). 

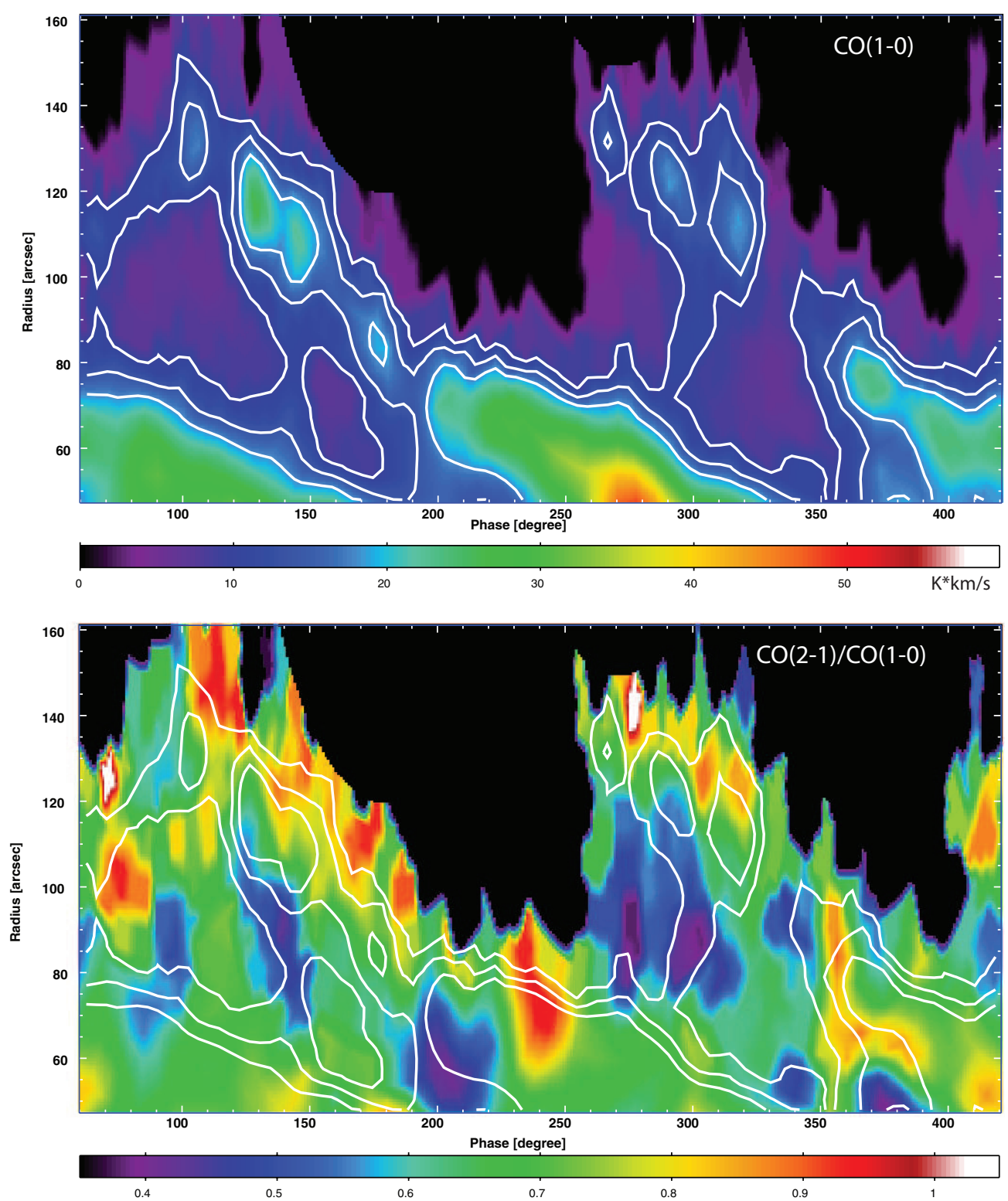

Figure 3. Phase diagrams of the $\mathrm{CO}(1-0)$ integrated intensity (top panel) and $R_{2-1 / 1-0}$ with $\mathrm{CO}(1-0)$ contours (bottom). The azimuthal angle (phase) is defined counterclockwise from the west. The plot spans over the full $360 \mathrm{deg}$, starting from $60 \mathrm{deg}$ to cover the two spiral arms without phase wraps. The counterclockwise gas flow in Figure 1 is toward the right in these diagrams. The gas with high $R_{2-1 / 1-0}$ appears predominantly at the downstream edge/side of the molecular spiral arms.

(A color version of this figure is available in the online journal.)

The spatial variations of $R_{2-1 / 1-0}$ across spiral arms are also confirmed in Figure 4. We adopt a simple logarithmic spiral pattern with a pitch angle of $20 \mathrm{deg}$ and measure statistical quantities around the spiral curves over 40 deg segments ( $20 \mathrm{deg}$ increment). The average $R_{2-1 / 1-0}$ increases to $>0.7$ in the molecular spiral arms (blue), especially on/near their leading edges (red), and decreases to $<0.7$ in the interarm regions. The areal fraction of high-ratio gas $(0.8-1.0)$ is high in the spiral arms and their leading edges, but low in the interarm regions. The fraction of low-ratio gas (0.4-0.6) is low in the arms, but high in the interarm regions. The central region also shows a high ratio. The average is 0.78 within the central $90^{\prime \prime}(\sim 3.6 \mathrm{kpc})$ region and 0.82 within $70^{\prime \prime}(\sim 2.8 \mathrm{kpc})$. (Example histograms of $R_{2-1 / 1-0}$ in the regions are presented in Appendix A and confirm the significant difference among the regions.)

Figure 2(a) shows the distribution of gas with low $\left(R_{2-1 / 1-0}=\right.$ $0.4-0.6)$, medium (0.6-0.8), and high ratios $(0.8-1.0)$ with respect to the molecular spiral arms (contours). Figure 2(b) highlights the distribution of the high ratios $(0.8-1.0)$. For reference, this figure includes the outer areas where a large number of pixels $(>10)$ need to be averaged. The variations of $R_{2-1 / 1-0}$ on large scales are evident: high ratios around the molecular spiral arms (contours), as well as in the center, and lower ratios in the interarm regions. The high ratios appear mostly at the leading edge/side of the molecular spiral arms, where OB stars are predominantly located (Figures 2(c) and (d)).

\subsection{Correlations}

Figure 5 shows pixel-by-pixel correlations of $R_{2-1 / 1-0}$ with three parameters (for the pixels in Figure 1). The $24 \mu \mathrm{m}$ surface brightness $f_{24 \mu \mathrm{m}}$ is roughly proportional to the star formation rate surface density (Calzetti et al. 2007), and the $\mathrm{CO}(1-0)$ integrated intensity $I_{\mathrm{CO}(1-0)}$ is proportional to the molecular gas surface density. Their ratio $f_{24 \mu \mathrm{m}} / I_{\mathrm{CO}(1-0)}$ is a measure of the star formation efficiency. Note that Calzetti et al. (2007) 

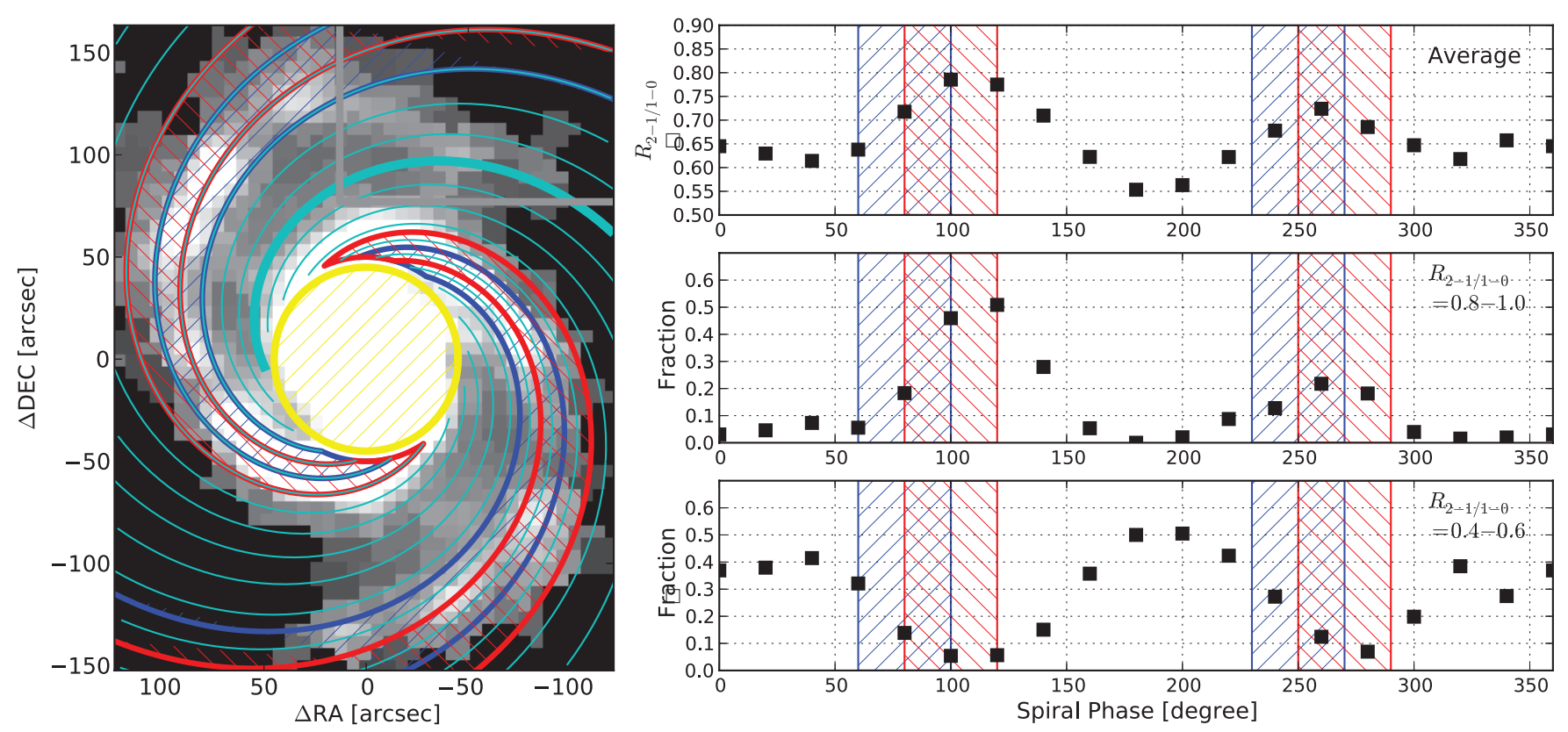

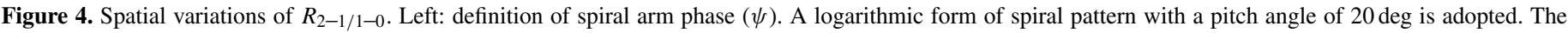

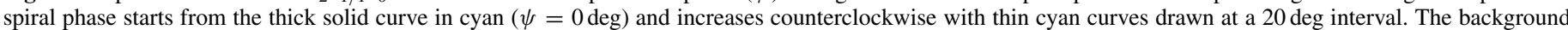

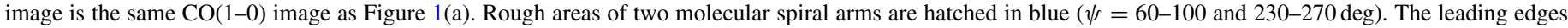

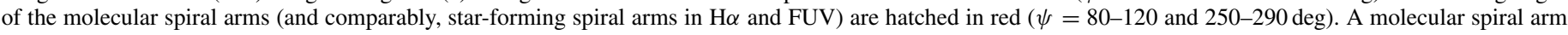

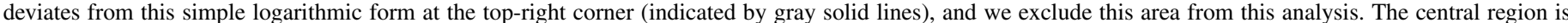

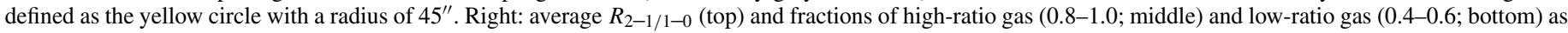

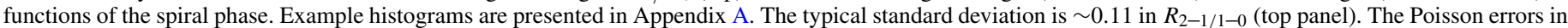

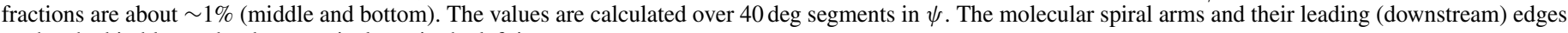
are hatched in blue and red, respectively, as in the left image.

(A color version of this figure is available in the online journal.)

discussed that the combination of $24 \mu \mathrm{m}$ and $\mathrm{H} \alpha$ emission provides a more accurate star formation rate; however, $f_{24 \mu \mathrm{m}}$ is used here as an approximate tracer, since it shows inherent correlations. Correlations with some other star formation rate tracers are given in Appendix B.

Most remarkable are the lower envelopes, or cutoffs, of the distributions in Figure 5 (left panels). No data points exist in the lower-right corners. We note that there are some dependences between $x$ and $y$ axes in the middle and bottom panels (though not in the top panels) as both axes include $I_{\mathrm{CO}(1-0)}$. However, we conclude that the cutoffs in all panels are intrinsic to physical conditions of the gas, since our detection limits cover the entire areas in these plots. Hence, $R_{2-1 / 1-0}$ increases with the intensity of star formation and gas surface density.

The right panels show correlations using averages and their scatter. $R_{2-1 / 1-0}$ increases clearly with star formation activities (i.e., $f_{24 \mu \mathrm{m}}$ and $\left.f_{24 \mu \mathrm{m}} / I_{\mathrm{CO}(1-0)}\right)$ and weakly with the gas surface density $\left(I_{\mathrm{CO}(1-0)}\right)$. The weaker correlation is due to the spatial offset between the molecular spiral arm and high $R_{2-1 / 1-0}$ (Section 3.1). Multiple physical processes may contribute to the intrinsic scatters in the left panels, and therefore linear model fits may be inappropriate. For instance, $R_{2-1 / 1-0}$ can be enhanced due to increases in density prior to star formation and increases in gas temperature due to heating after star formation (Section 4).

In addition to the clear correlations with star formation activities, some high values of $R_{2-1 / 1-0}$ are seen with low $f_{24 \mu \mathrm{m}}$, $I_{\mathrm{CO}(1-0)}$, and $f_{24 \mu \mathrm{m}} / I_{\mathrm{CO}(1-0)}$. Figure 6 shows the locations of this component, i.e., with a high ratio, $R_{2-1 / 1-0}>0.8$, and low star formation efficiency, $f_{24 \mu \mathrm{m}} / I_{\mathrm{CO}(1-0)}<0.6$, with respect to $f_{24 \mu \mathrm{m}}$ (white contours) and $\mathrm{H} \alpha$ emission (right panel). Some regions with the elevated ratios appear at the upstream edges of the $24 \mu \mathrm{m}$ spiral arm, possibly indicating dense pre-OB star formation gas (see Section 4).

\section{DISCUSSION}

GMCs are abundant even in the interarm regions in M51 (Koda et al. 2009). Although our 19!'7 ( 780 pc) beam typically contains many GMCs, we adopt a one-zone approximation to obtain insights into the mean physical conditions of the molecular gas and its spatial variations. That is, we assume that the GMCs within a resolution element share the same average properties and that $\mathrm{CO}(1-0)$ and $\mathrm{CO}(2-1)$ are emitted from the same physical regions.

\subsection{Large Velocity Gradient Analysis}

The large velocity gradient (LVG) approximation is often used for the line radiation transfer of interstellar molecular lines (Scoville \& Solomon 1974; Goldreich \& Kwan 1974). This approach enables treatment of the coupled radiative transfer and molecular excitation as a local problem since the line emission in different volumes of gas is Doppler shifted and decoupled; the approach is justified by the fact that thermal line broadening $\left(\sim 0.2-0.3 \mathrm{~km} \mathrm{~s}^{-1}\right)$ is much smaller than velocity dispersions within GMCs ( $\gtrsim$ a few $\mathrm{km} \mathrm{s}^{-1}$ ). The molecular excitation conditions then depend on the local kinetic temperature $T_{\text {kin }}$, volume density $n_{\mathrm{H}_{2}}$ (since low- $J \mathrm{CO}$ emission is excited collisionally), and local line opacity (i.e., $\mathrm{CO}$ column density $N_{\mathrm{CO}}$ per unit velocity change). Thus, $\left(T_{\mathrm{kin}}, n_{\mathrm{H}_{2}}, N_{\mathrm{CO}} / d v\right)$ determine the line ratio $R_{2-1 / 1-0}$, or conversely, an observed $R_{2-1 / 1-0}$ constrains these parameters, albeit with some degeneracy. We have developed our own LVG calculation code using the newest $\mathrm{CO}-\mathrm{H}_{2}$ collisional cross sections from Yang et al. (2010). 

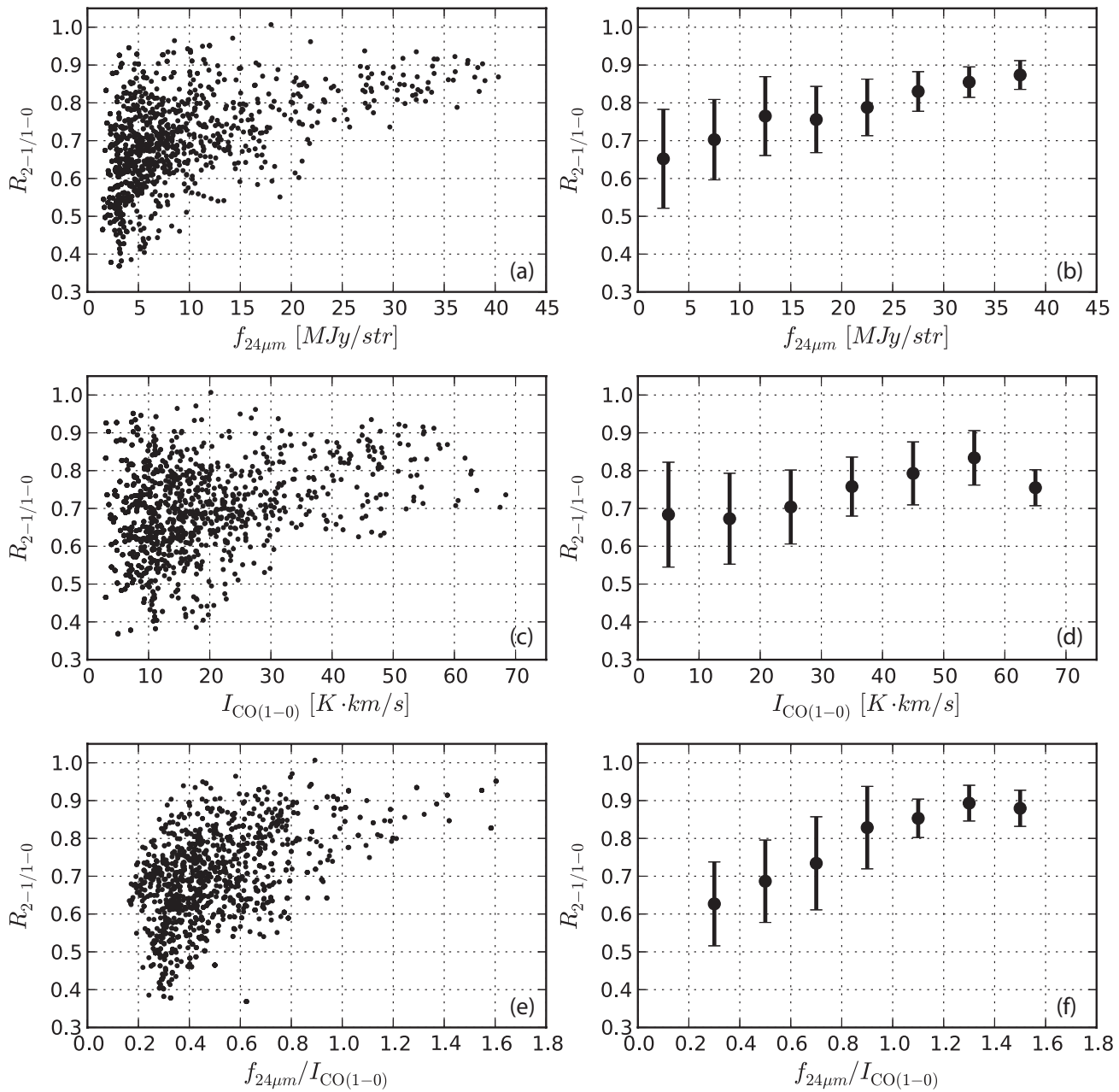

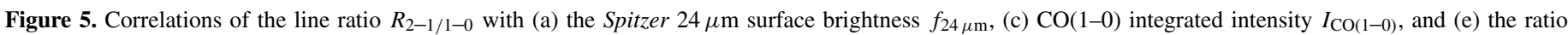
of $f_{24 \mu \mathrm{m}}$ and $I_{\mathrm{CO}(1-0)}$. (b), (d), and (f) are the same as (a), (c), and (e), respectively, but with binned averages and standard deviations. The $f_{24 \mu \mathrm{m}}, I_{\mathrm{CO}(1-0)}$, and

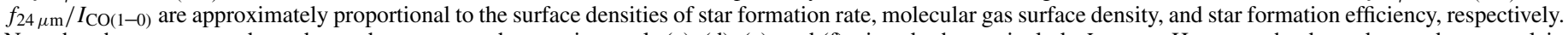
Note that there are some dependences between $x$ and $y$ axes in panels (c), (d), (e), and (f), since both axes include $I_{\mathrm{CO}(1-0)}$. However, the dependences do not explain the absence of data in the lower-right corners, since the detected parameter ranges can cover the corners.

The CO column density per unit velocity gradient is typically in the range $\log \left(N_{\mathrm{CO}} / d v\left(\mathrm{~cm}^{-2}\left(\mathrm{~km} \mathrm{~s}^{-1}\right)^{-1}\right)\right)=16.6-17.3$ based on the observed line widths and assuming a standard ISM $\mathrm{CO} / \mathrm{H}_{2}$ abundance of $8 \times 10^{-5}$ (Schinnerer et al. 2010). For example, Galactic GMCs have approximately constant mean surface densities averaged over their entire areas $\left(\sim 170 M_{\odot} \mathrm{pc}^{-2}\right.$; Solomon et al. 1987; Rodriguez-Fernandez et al. 2006) and a range of velocity widths $\sim 4-18 \mathrm{~km} \mathrm{~s}^{-1}$, resulting in the above range of $N_{\mathrm{CO}} / d v$. For the most extreme surface densities in M51's spiral arms, the mass surface density is $\sim 1000 M_{\odot} \mathrm{pc}^{-2}$, while the velocity width is $50-100 \mathrm{~km} \mathrm{~s}^{-1}$ (Koda et al. 2009), yielding a similar $N_{\mathrm{CO}} / d v$.

Figure 7 shows the parameter ranges for $T_{\text {kin }}$ and $n_{\mathrm{H}_{2}}$ which yield values of $R_{2-1 / 1-0}=0.4,0.6,0.8,0.9$ and 1.0. In each case, two curves are shown corresponding to $\log \left(N_{\mathrm{CO}} / d v\right)=$ 17.3 and 16.6. (Note that the relative position of the two curves is reversed for $R_{2-1 / 1-0} \geqslant 0.9$, compared to that for lower values of $R_{2-1 / 1-0}$.) Values of $R_{2-1 / 1-0}<0.4$ and $>1$ can be obtained but are not shown here since they are not seen in our observations. Galactic GMCs typically have $T_{\text {kin }} \sim 10 \mathrm{~K}$ and $n_{\mathrm{H}_{2}} \sim 300 \mathrm{~cm}^{-3}\left(\log n_{\mathrm{H}_{2}} \sim 2.5\right)$ with a scatter of about a factor of 2 (Scoville \& Sanders 1987; Solomon et al. 1987), yielding a range in $R_{2-1 / 1-0} \sim 0.4-0.8$ and a mean observed ratio of $0.6-0.7$ from Figure 7 . This range is consistent with the observed $R_{2-1 / 1-0}$ in interarm GMCs in the Galaxy and the bulk parts of the Orion GMCs, i.e., the larger region not closely associated with active high-mass star formation (Sakamoto et al. 1997).

Figure 7 shows that $R_{2-1 / 1-0}$ is a sensitive probe of physical conditions in the bulk molecular gas. A high value of $R_{2-1 / 1-0} \sim$ 0.8 requires increases in either $T_{\mathrm{kin}}$ or $n_{\mathrm{H}_{2}}$ (or both) by a factor of $\sim 2-3$, relative to the standard GMC values of $10 \mathrm{~K}$ and $300 \mathrm{~cm}^{-3}$. To reproduce the even higher ratios $\sim 0.9-1.0$ within an expected range of $T_{\mathrm{kin}}$ or $n_{\mathrm{H}_{2}}, T_{\mathrm{kin}}$ and $n_{\mathrm{H}_{2}}$ need to increase more than factors of $\sim 3$ and $\sim 10$, respectively.

$R_{2-1 / 1-0}$ shows spatial variations in M51. The ratio often reaches as low as $\sim 0.4-0.6$ in the interarm regions, while it becomes as high as $\sim 0.8-1.0$ in the spiral arms (Figures 1, 2, and 4$)$. The lower $R_{2-1 / 1-0}(\sim 0.4-0.6)$ generally seen in the interarm regions implies that the gas there has significantly lower $T_{\text {kin }}$ and $n_{\mathrm{H}_{2}}$ than that in the arms. Based on the similarity in $R_{2-1 / 1-0}$, we conclude that the GMCs in M51's interarm regions have typical physical conditions similar to their Galactic counterparts. In the Galaxy, the mass dividing line for $50 \%$ 

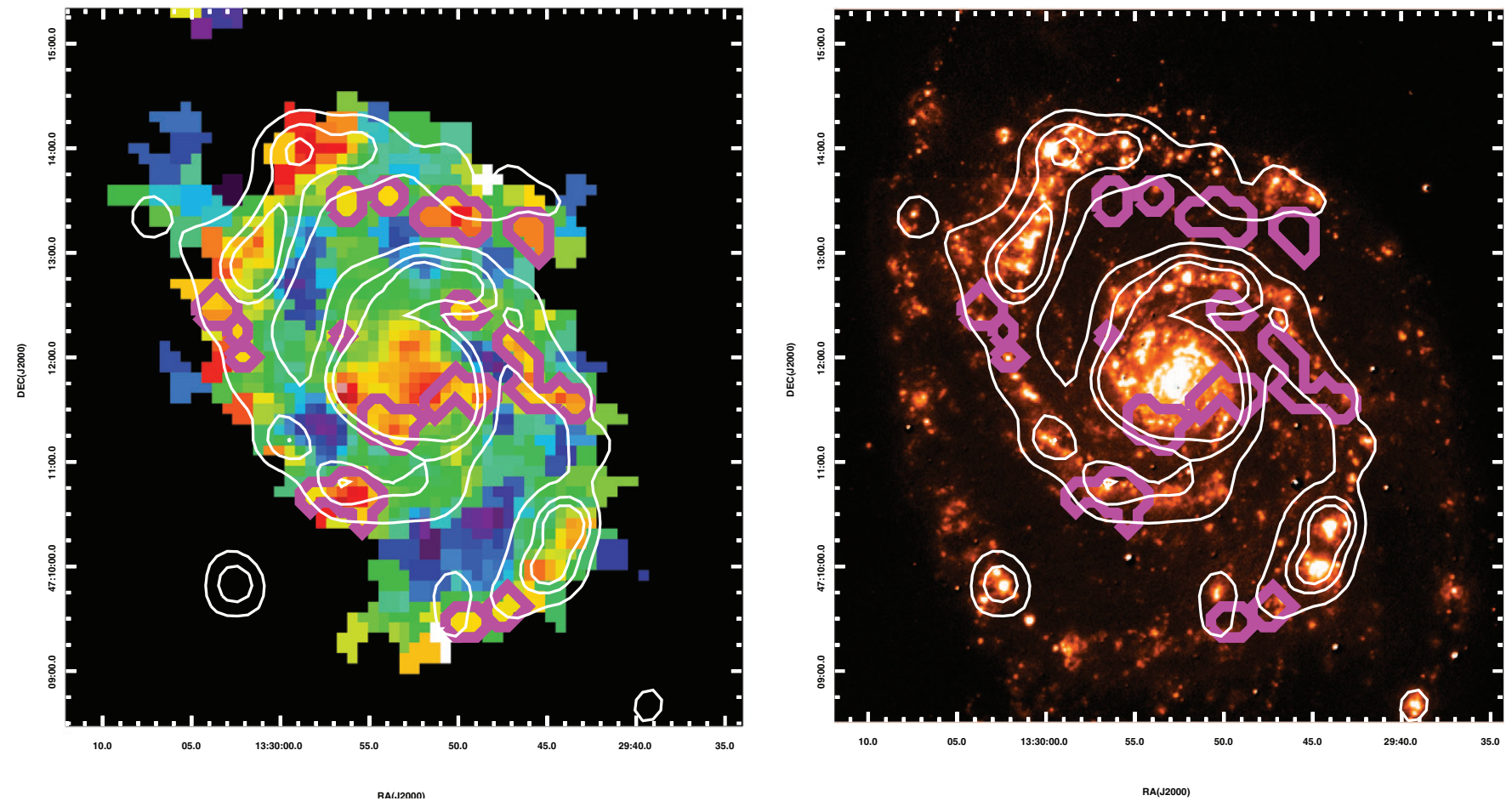

Figure 6. Comparisons of the $\mathrm{CO} J=2-1 / 1-0$ line ratio $R_{2-1 / 1-0}$ (left), $\mathrm{H} \alpha$ image (right), and contours (white) from the Spitzer $24 \mu \mathrm{m}$ image (without smoothing) at 4,8 , and $12 \mathrm{MJy} \mathrm{sr}^{-1}$. The color scale of the left panel is the same as that in Figure 1 . The high $R_{2-1 / 1-0}$ regions coincide mostly with star-forming regions (with high $f_{24 \mu \mathrm{m}}$ and $\mathrm{H}$ II regions). The contours in magenta are the regions of high $R_{2-1 / 1-0},>0.8$, and low star formation efficiency, $f_{24} \mu \mathrm{m} / I_{\mathrm{CO}(1-0)}<0.6$ (see Figure 5) These regions appear on both the spiral arms and their trailing/upstream edges (e.g., in the northwest quadrant), possibly indicating the growth of pre-star-forming dense cores.

(A color version of this figure is available in the online journal.)

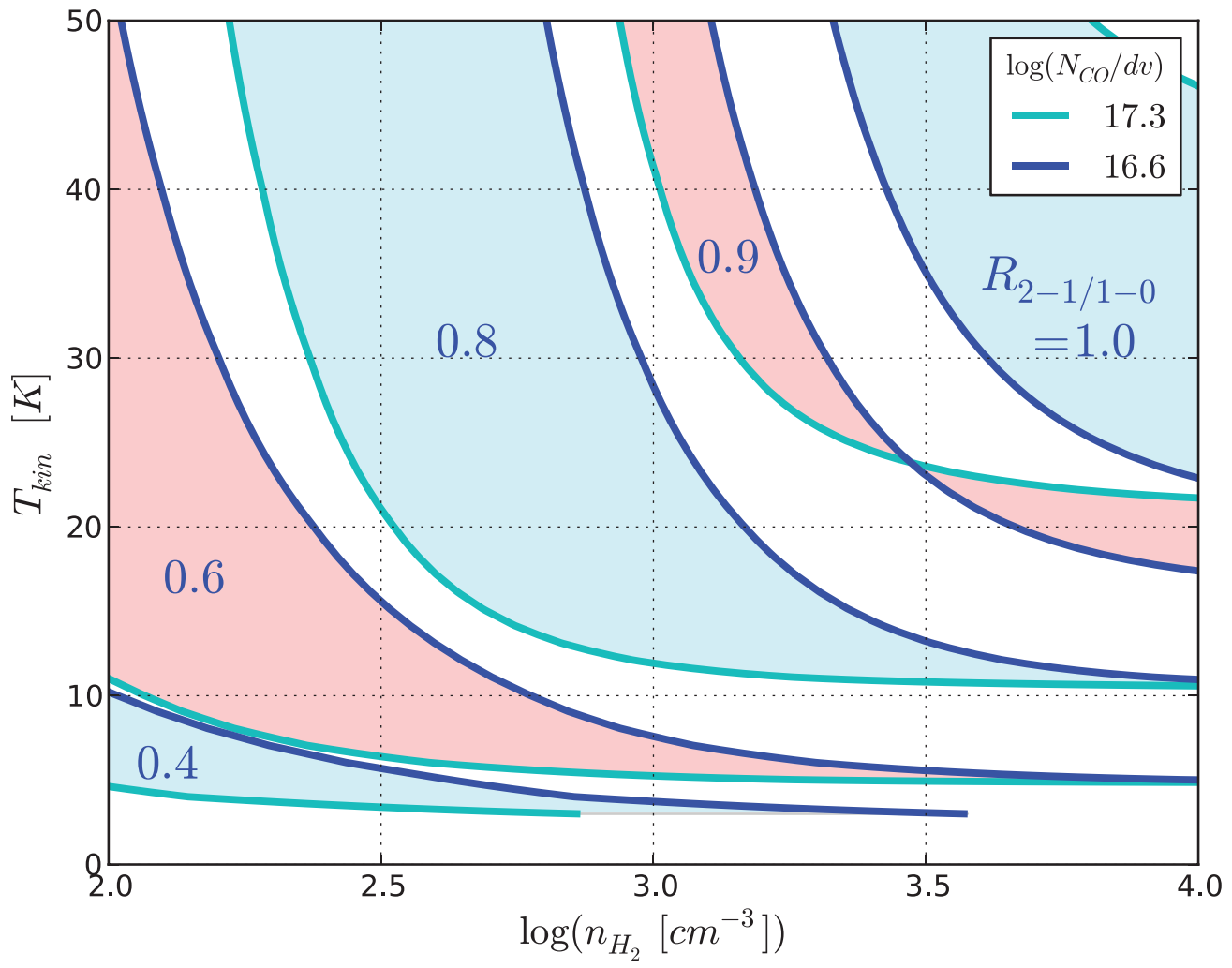

Figure 7. LVG calculations. The line ratios $R_{2-1 / 1-0}$ are plotted as functions of the gas kinetic temperature $T_{\text {kin }}$ and $\mathrm{H}_{2}$ density $n_{\mathrm{H}_{2}}$. Most GMCs are in the range of the $\mathrm{CO}$ column densities $\log \left(N_{\mathrm{CO}} / d v\right)=16.6$ (blue) and 17.3 (cyan) assuming the $\mathrm{CO}$ fractional abundance to $\mathrm{H}_{2}$ of $8 \times 10^{-5}$. The regions between the curves for the two column densities (blue and cyan) are shaded for clarity.

(A color version of this figure is available in the online journal.) 
of the total $\mathrm{H}_{2}$ content is $\sim 4 \times 10^{5} M_{\odot}$, i.e., GMCs more massive than this account for half the total molecular gas mass (Scoville \& Sanders 1987). In M51, we can derive a similar GMC mass in the 50th percentile of $\sim 5 \times 10^{5} M_{\odot}$ using higher resolution interferometric imaging (Koda et al. 2009, 2011), which is capable of resolving the emission of more massive clouds and comparing their total emission with that in the singledish maps, which also include contributions from lower mass clouds.

The higher $R_{2-1 / 1-0} \sim 0.8-1.0$, seen typically on the leading (downstream) edges of the spiral arms, requires higher values, in both $T_{\text {kin }}$ and $n_{\mathrm{H}_{2}}$ by a factor of 2-3, compared to those of typical GMCs and interarm GMCs. This downstream gas is associated with ongoing star formation (Section 3). Therefore, increases in both $T_{\text {kin }}$ and $n_{\mathrm{H}_{2}}$, rather than in either $T_{\text {kin }}$ or $n_{\mathrm{H}_{2}}$ alone, may be more likely. On the other hand, the gas with high $R_{2-1 / 1-0} \sim$ $0.8-1.0$ on the upstream edge typically shows low star formation activity (Figure 6), possibly explained by increases in $n_{\mathrm{H}_{2}}$ alone, without significant increase in $T_{\text {kin }}$.

Of course, this one-zone analysis is simplistic for regions extended over $\sim 780 \mathrm{pc}$, but it still provides an indication that the bulk properties of the gas evolve from the interarm regions through the spiral arms. The interarm gas is mostly in GMCs without active star formation, and upon entry into the spiral arms, the molecular gas becomes warmer and/or denser by factors of at least $\sim 2-3$, possibly due to dynamical compression (e.g., at the upstream edges), but primarily due to young stellar heating (at the downstream edges).

\subsection{Comparison with Galactic Studies}

A similar evolution of molecular gas, i.e., warmer and/or denser in spiral arms, has been observed in the Galaxy, although uncertainties remain due to our in-plane viewpoint for the Milky Way. Sanders et al. (1985) found GMCs with high brightness temperature mostly associated with spiral arms as delineated by radio HII regions. Sawada et al. (2012a, 2012 b) recently demonstrated that the molecular gas with high brightness temperature is located continuously along the spiral arms in the longitude-velocity diagram. Sakamoto et al. $(1995,1997)$ found, from their Galactic Plane CO(2-1) survey, that molecular gas with the highest $R_{2-1 / 1-0}$ is predominantly along the Sagittarius and Scutum spiral arms. Both the Galaxy and M51 exhibit similar variation in $R_{2-1 / 1-0}$ across their disks.

The great advantage of the Galactic studies is their high linear resolution. Sakamoto et al. (1997) and Hasegawa (1997) classified molecular gas into three categories based on their $R_{2-1 / 1-0}$ measurements: low-ratio gas $\left(R_{2-1 / 1-0}<0.7\right)$, highratio gas $(0.7-1.0)$, and very high ratio gas $(>1.0)$. The low-ratio gas is often observed in large, non-star-forming parts of GMCs (Sakamoto et al. 1994) and in interarm GMCs (Sakamoto et al. 1997). The very high ratio gas appears exclusively immediately around H II regions (e.g., the M42 Nebula; Sakamoto et al. 1994) and hence is likely strongly heated by radiation at the boundary of the $\mathrm{H}$ II regions. The high-ratio gas can also be found around the $\mathrm{H}$ II regions, just outside the very high ratio gas. In Galactic GMCs, high ratios are also associated with dense core regions prior to efficient star formation, but where the $\mathrm{CO}$ levels have become fully thermalized and the temperatures are modestly elevated (Hasegawa 1997).

The central $\sim 2.8 \mathrm{kpc}$ region of M51 shows high $R_{2-1 / 1-0}(\sim 0.8-1.0)$. This is similar to the Galactic center, where $R_{2-1 / 1-0} \sim 1.0$, and sometimes $>1$, over its central
900 pc region. These ratios are quite high in comparison with typical GMC values, though they are around the average for galactic centers (some starburst galaxies show $R_{2-1 / 1-0}>1.0$ in their centers; Braine \& Combes 1992; Sawada et al. 2001). Sawada et al. (2001) explain that the high and very high ratios in the Galactic center are caused by intrinsically high $T_{\text {kin }}$ and $n_{\mathrm{H}_{2}}$, combined with low opacity in low- $J$ transitions due to the large velocity dispersion and a very high excitation $(J \geqslant 3)$. The high ratios (0.8-1.0) in the center of M51 probably indicate similar physical conditions to those of the Galactic Central Molecular Zone.

\subsection{Causes of High Ratios}

The spiral arm and galactic nucleus GMCs with higher $R_{2-1 / 1-0}$ are likely heated primarily by associated OB star formation (Figures 1, 2, and 5), and their densities raised by dynamical compression within the spiral arms (i.e., close encounters and/or collisions with other GMCs) and shearing (Koda et al. 2009), all of which can lead to the high-ratio gas ( $>0.7$, and up to $0.8-1.0)$.

A substantial fraction of ionizing photons leaking out of $\mathrm{H}$ II regions (Ferguson et al. 1996; Wang et al. 1999; Blanc et al. 2009) might be a cause of the elevated heating outside the $\mathrm{H}$ II regions. However, to alter the temperatures over the very large regions ( $\gtrsim 780 \mathrm{pc}$ ) at the leading sides of the spiral arms (as seen here), it seems more likely that the radiative heating is provided by the longer wavelength continuum of the young star clusters (i.e., longward of the Lyman limit) combined with hydrogen recombination line radiation from the $\mathrm{H}$ II regions (since the Lyman continuum is almost surely absorbed over these long paths). An abundant population of active $\mathrm{H}$ II regions exists along the spiral arms, some of them 10-100 times brighter than the Orion Nebula (Scoville et al. 2001), and of course there is considerable luminosity longward of the Lyman limit. High ratios over a large area are also found around the 30 Doradus complex in the Large Magellanic Cloud, one of the most active star-forming regions in the Local Group (Sorai et al. 2001).

We note that cloud-cloud tidal interactions within the spiral arms can also inject a large amount of internal energy into GMCs in very close encounters. The typical gas thermal energy in a GMC is $\sim 2 \times 10^{47} \mathrm{erg}$; this is two orders of magnitude smaller than the energy increase due to a close cloud-cloud encounter, $\sim 3 \times 10^{49}$ erg (estimated using the impulse approximation (Spitzer 1958), an encounter speed of $10 \mathrm{~km} \mathrm{~s}^{-1}$, and typical GMC parameters (Scoville \& Sanders 1987)). Dynamical environments in the spiral arms can possibly explain high ratios at the spiral arm entry (at their upstream edges).

\section{IMPLICATIONS TO OTHER STUDIES}

Our results indicate that $\mathrm{CO}(2-1)$ emission is sensitive to the change of physical conditions of bulk molecular gas in GMCs, in contrast to $\mathrm{CO}(1-0)$, which traces the bulk molecular mass. The spatial variation of $R_{2-1 / 1-0}$ indicates the evolution in physical conditions of the gas as it passes from interarm to arm regions during the course of azimuthal orbital motion. The use of $\mathrm{CO}(2-1)$ is reasonable as a tracer of molecular gas mass when galactic structures are unresolved, e.g., in studies of highredshift galaxies (e.g., Frayer et al. 2008), since the $R_{2-1 / 1-0}$ varies only by a factor of $\sim 2$. However, spatially resolved studies of $\mathrm{CO}(2-1)$ in nearby galaxies may be quite misleading with 
respect to inferred variations in the gas mass surface density due to changes in $R_{2-1 / 1-0}$ with both radius (e.g., in the nuclear regions) and azimuth due to the spiral arms. The systematic trends illustrated in Figure 5 will affect correlation studies of the gas mass and other parameters such as the Schmidt relation (Bigiel et al. 2008; Leroy et al. 2008; Schruba et al. 2011). In fact, the Schmidt relation using the CANON CO(1-0) data of $\sim 10$ galaxies shows a nonlinear, power-law relationship (Liu et al. 2011; R. Momose et al. 2012, in preparation), as opposed to the linear relation derived from the above-quoted $\mathrm{CO}(2-1)$ studies.

\section{SUMMARY}

New multi-beam receivers and OTF mapping technique in millimeter astronomy enable us to map the large molecular disk of M51 in CO $J=1-0$ and 2-1 transitions with unprecedented accuracy. We find systematic spatial variations in the line ratio $R_{2-1 / 1-0}$-from low $(<0.7$, often $\sim 0.4-0.6)$ in the interarm regions to high $(>0.7, \sim 0.8-1.0)$ in the spiral arms, especially on their leading (downstream) edge. The central $\sim 2.8 \mathrm{kpc}$ region also exhibits high $R_{2-1 / 1-0}(\sim 0.8-1.0)$.

The interarm gas is mostly in GMCs without active star formation, and upon entry into the spiral arms, the molecular gas becomes warmer and/or denser by factors of $\sim 2-3$. The gas in the spiral arms and galactic nucleus are likely heated primarily by associated OB star formation (especially their continuum radiation longward of the Lyman limit) and their densities raised by dynamical compression within the spiral arms. Some high-ratio gas exists on the upstream edges of the spiral arms, perhaps indicating gas becoming dense prior to star formation. Similar variations in the $\mathrm{CO}$ line ratios have been seen in the Galaxy-GMCs with inactive star formation have low $R_{2-1 / 1-0}(\sim 0.4-0.6)$, and ones with active star formation show high $R_{2-1 / 1-0}(\sim 0.8-1.0)$.

The Nobeyama $45 \mathrm{~m}$ telescope is operated by the Nobeyama Radio Observatory, a branch of the National Astronomical Observatory of Japan. We thank Seiichi Sakamoto and Jim Barrett for valuable comments. We also thank the anonymous referee for valuable comments. J.K. acknowledges support from the NSF through grant AST-1211680 and NASA through grant NNX09AF40G, a Hubble Space Telescope grant, and a Herschel Space Observatory grant.

\section{APPENDIX A \\ HISTOGRAMS OF $R_{2-1 / 1-0}$}

Figure 8 presents sample histograms of $R_{2-1 / 1-0}$ in spiral arms, interarm regions, the entire disk, and the central region defined in Figure 4. Differences in the average $R_{2-1 / 1-0}$ between the spiral arms and interarm regions are significant compared to the standard deviations.

The average ratio of the spiral arm of $260 \mathrm{deg}$ is smaller than that of $100 \mathrm{deg}$. The fraction of high-ratio gas is also lower for the $260 \mathrm{deg}$ arm (Figure 4), though the increase from interarm regions is still evident. These differences are partly due to the high-ratio gas appearing upstream this spiral arm at smaller radii and downstream at larger radii (Figures 1 and 2). A full discussion of the nature of this spatial variation (upstream vs. downstream) is beyond the scope of this paper, but possible causes include the scattered distribution of star-forming regions along this arm (Egusa et al. 2009), cloud-cloud interactions, or dynamical gas compression due to the wiggle instability (Wada \& Koda 2004).

\section{APPENDIX B}

\section{CORRELATIONS BETWEEN $R_{2-1 / 1-0}$ AND STAR FORMATION RATE TRACERS}

We adopted the $24 \mu \mathrm{m}$ image as an approximate tracer of star formation rate and showed the correlation between $R_{2-1 / 1-0}$ and star formation activities in Section 3.2. Similar correlations exist with other tracers of star formation rate. Figure 9 shows correlations with $\mathrm{H} \alpha$ flux and with star formation rate calculated by combining $24 \mu \mathrm{m}$ and $\mathrm{H} \alpha$ (Calzetti et al. 2007; Kennicutt et al. 2007).
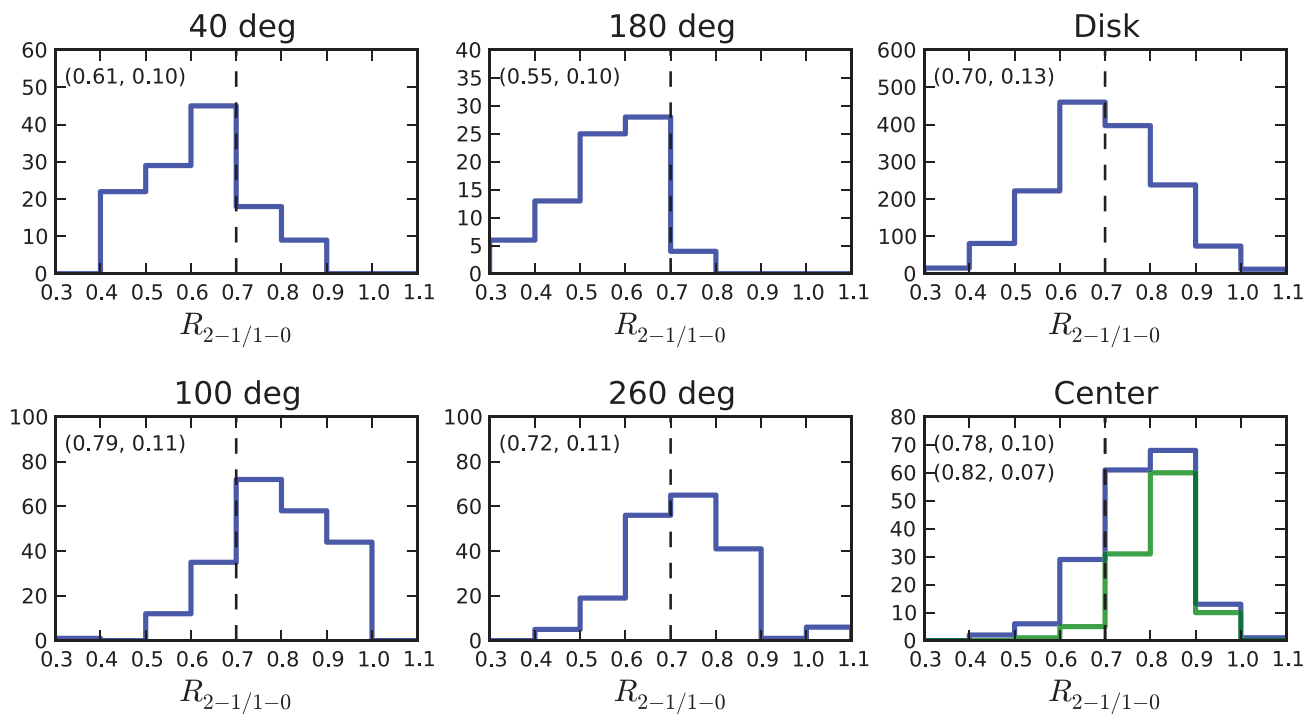

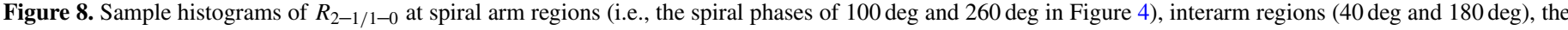

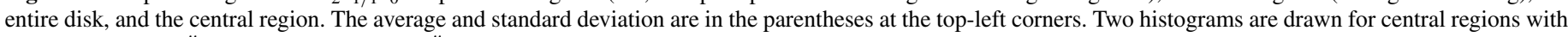
the diameters of $90^{\prime \prime}$ ( $~ 3.6 \mathrm{kpc}$; blue) and 70" ( $2.8 \mathrm{kpc}$; green).

(A color version of this figure is available in the online journal.) 

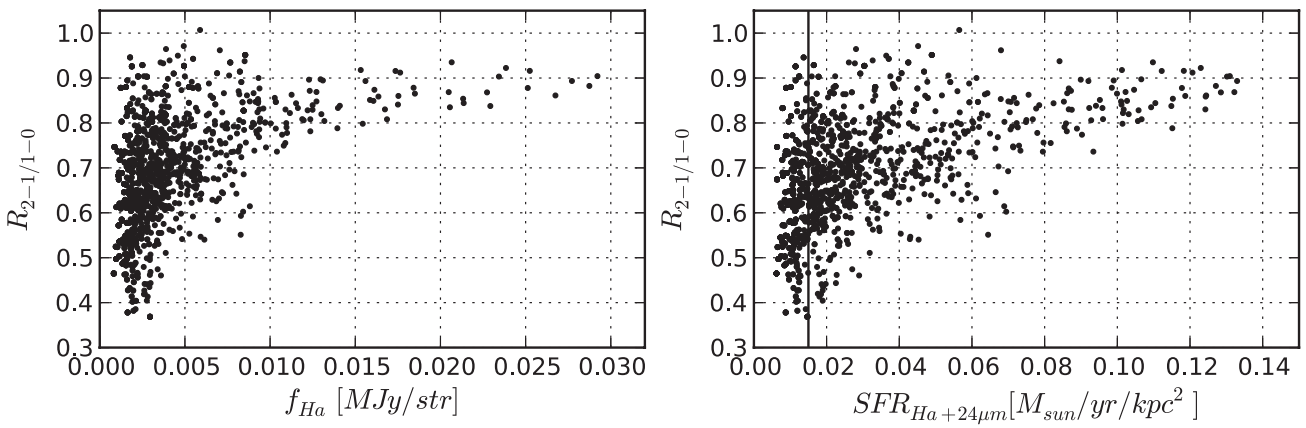

Figure 9. $R_{2-1 / 1-0}$ as functions of $\mathrm{H} \alpha$ emission (left) and star formation rate (SFR) (right) calculated with $24 \mu \mathrm{m}$ and $\mathrm{H} \alpha$ emission (Calzetti et al. 2007; Kennicutt et al. 2007). The solid vertical line in the right panel indicates a boundary below which the background emission unrelated to recent star formation may contaminate the measurement (Calzetti et al. 2007; Liu et al. 2011).

\section{REFERENCES}

Aalto, S., Booth, R. S., Black, J. H., \& Johansson, L. E. B. 1995, A\&A, 300,369

Bigiel, F., Leroy, A., Walter, F., et al. 2008, AJ, 136, 2846

Blanc, G. A., Heiderman, A., Gebhardt, K., Evans, N. J., II, \& Adams, J. 2009, ApJ, 704, 842

Bolatto, A. D., Leroy, A. K., Rosolowsky, E., Walter, F., \& Blitz, L. 2008, ApJ, 686, 948

Braine, J., \& Combes, F. 1992, A\&A, 264, 433

Calzetti, D., Kennicutt, R. C., Engelbracht, C. W., et al. 2007, ApJ, 666, 870

Cappellari, M., \& Copin, Y. 2003, MNRAS, 342, 345

Donovan Meyer, J., Koda, J., Momose, R., et al. 2012, ApJ, 744, 42

Egusa, F., Koda, J., \& Scoville, N. 2011, ApJ, 726, 85

Egusa, F., Kohno, K., Sofue, Y., Nakanishi, H., \& Komugi, S. 2009, ApJ, 697, 1870

Ferguson, A. M. N., Wyse, R. F. G., Gallagher, J. S., III., \& Hunter, D. A. 1996, AJ, 111, 2265

Frayer, D. T., Koda, J., Pope, A., et al. 2008, ApJ, 680, L21

Gao, Y., \& Solomon, P. M. 2004, ApJ, 606, 271

Garcia-Burillo, S., Guelin, M., \& Cernicharo, J. 1993, A\&A, 274, 123

Gil de Paz, A., Boissier, S., Madore, B. F., et al. 2007, ApJS, 173, 185

Goldreich, P., \& Kwan, J. 1974, ApJ, 189, 441

Grenier, I. A., Casandjian, J.-M., \& Terrier, R. 2005, Science, 307, 1292

Hagiwara, Y., Henkel, C., Menten, K. M., \& Nakai, N. 2001, ApJ, 560, L37

Harrison, A., Henkel, C., \& Russell, A. 1999, MNRAS, 303, 157

Hasegawa, T. 1997, in IAU Symp. 170, The CO 2-1/1-0 Ratio, ed. W. B.

Latter, S. J. E. Radford, P. R. Jewell, J. G. Mangum, \& J. Bally (Cambridge: Cambridge Univ. Press), 39

Israel, F. P. 1997, A\&A, 328, 471

Kennicutt, R. C., Jr., Armus, L., Bendo, G., et al. 2003, PASP, 115, 928

Kennicutt, R. C., Jr., Calzetti, D., Walter, F., et al. 2007, ApJ, 671, 333

Knapp, G. R., Leighton, R. B., Wannier, P. G., Phillips, T. G., \& Huggins, P. J. 1980, ApJ, 240, 60

Koda, J., Sawada, T., Wright, M. C. H., et al. 2011, ApJS, 193, 19

Koda, J., Scoville, N., Sawada, T., et al. 2009, ApJ, 700, L132

Kohno, K., Kawabe, R., Tosaki, T., \& Okumura, S. K. 1996, ApJ, 461, L29

Komugi, S., Kohno, K., Tosaki, T., et al. 2007, PASJ, 59, 55

Leroy, A. K., Bolatto, A., Bot, C., et al. 2009a, ApJ, 702, 352

Leroy, A. K., Walter, F., Bigiel, F., et al. 2009b, AJ, 137, 4670

Leroy, A. K., Walter, F., Brinks, E., et al. 2008, AJ, 136, 2782

Liu, G., Koda, J., Calzetti, D., Fukuhara, M., \& Momose, R. 2011, ApJ, 735, 63
Muraoka, K., Kohno, K., Tosaki, T., et al. 2007, PASJ, 59, 43

Nakai, N., Kuno, N., Handa, T., \& Sofue, Y. 1994, PASJ, 46, 527

Planck Collaboration, Ade, P. A. R., Aghanim, N., et al. 2011, A\&A, 536, A19

Rodriguez-Fernandez, N. J., Braine, J., Brouillet, N., \& Combes, F. 2006, A\&A, 453,77

Sakamoto, S., Hasegawa, T., Handa, T., Hayashi, M., \& Oka, T. 1997, ApJ, 486,276

Sakamoto, S., Hasegawa, T., Handa, T., et al. 1999, in ASP Conf. Ser. 168, New Perspectives on the Interstellar Medium, ed. A. R. Taylor, T. L. Landecker, \& G. Joncas (San Francisco, CA: ASP), 90

Sakamoto, S., Hasegawa, T., Hayashi, M., Handa, T., \& Oka, T. 1995, ApJS, 100,125

Sakamoto, S., Hayashi, M., Hasegawa, T., Handa, T., \& Oka, T. 1994, ApJ, 425,641

Sanders, D. B., Scoville, N. Z., \& Solomon, P. M. 1985, ApJ, 289, 373

Sawada, T., Hasegawa, T., Handa, T., et al. 2001, ApJS, 136, 189

Sawada, T., Hasegawa, T., \& Koda, J. 2012a, ApJ, 759, L26

Sawada, T., Hasegawa, T., Sugimoto, M., Koda, J., \& Handa, T. 2012b, ApJ, 752,118

Sawada, T., Ikeda, N., Sunada, K., et al. 2008, PASJ, 60, 445

Schinnerer, E., Weiß, A., Aalto, S., \& Scoville, N. Z. 2010, ApJ, 719,1588

Schruba, A., Leroy, A. K., Walter, F., et al. 2011, AJ, 142, 37

Schuster, K. F., Kramer, C., Hitschfeld, M., Garcia-Burillo, S., \& Mookerjea, B. 2007, A\&A, 461, 143

Scoville, N. Z., Polletta, M., Ewald, S., et al. 2001, AJ, 122, 3017

Scoville, N. Z., \& Sanders, D. B. 1987, in Astrophysics and Space Science Library, Vol. 134, Interstellar Processes, ed. D. J. Hollenbach \& H. A. Thronson, Jr. (Dordrecht: Reidel), 21

Scoville, N. Z., \& Solomon, P. M. 1974, ApJ, 187, L67

Solomon, P. M., Rivolo, A. R., Barrett, J., \& Yahil, A. 1987, ApJ, 319, 730

Sorai, K., Hasegawa, T., Booth, R. S., et al. 2001, ApJ, 551, 794

Spitzer, L., Jr. 1958, ApJ, 127, 17

Sunada, K. Yamaguchi, C., Nakai, N., et al. 2000, Proc. SPIE, 4015, 237

Turner, J. L., Hurt, R. L., \& Hudson, D. Y. 1993, ApJ, 413, L19

Wada, K., \& Koda, J. 2004, MNRAS, 349, 270

Wang, J., Heckman, T. M., \& Lehnert, M. D. 1999, ApJ, 515, 97

Wilson, C. D., Warren, B. E., Israel, F. P., et al. 2009, ApJ, 693, 1736

Wu, J., Evans, N. J., II, Gao, Y., et al. 2005, ApJ, 635, L173

Yang, B., Stancil, P. C., Balakrishnan, N., \& Forrey, R. C. 2010, ApJ, 718, 1062 